\title{
Classificação de Transformações Conformes da Esfera para o Plano
}

\author{
L. BOURCHTEIN 1 , A. BOURCHTEIN ${ }^{2}$, E. OLIVEIRA, B. HERREIRA, \\ Departamento de Matemática, Universidade Federal de Pelotas, RS, Brasil.
}

Resumo. Neste trabalho desenvolvemos uma abordagem alternativa a geração de aplicações conformes da esfera para o plano. No conjunto das projeções consideradas são incluídas as estereográficas, cônicas e cilíndricas, que são as mais usadas em modelagem atmosférica e oceânica. Introduzindo coeficiente de variação do fator de mapa como medida do grau de homogeneidade de grades computacionais, utilizamos a abordagem proposta para classificar as aplicações consideradas e determinar a sua equivalência à classe de projeções cujo fator de mapa não depende da longitude.

\section{Introdução}

Muitos processos da dinâmica da atmosfera e oceano são de grande escala o que implica a formulação de modelos matemáticos em coordenadas esféricas. Devido a complexidade dos modelos, a sua solução analítica é desconhecida e as soluções aproximadas podem ser encontradas pela aplicação de métodos numéricos. A utilização de coordenadas esféricas na geração de grades computacionais leva ao espaçamento físico altamente não homogêneo, o que gera problemas tanto para parte dinâmica como para a física dos modelos numéricos. A abordagem alternativa mais usada é a aplicação conforme da esfera para o plano com posterior geração de grades em coordenadas planares. Este método permite manter a forma mais simples das equações diferenciais primitivas e garante o tratamento localmente isotrópico das derivadas e a suavidade de variação do espaçamento físico. As projeções conformes mais usadas são estereográficas, cônicas e cilíndricas. As primeiras duas, normalmente, se aplicam nas regiões de latitudes altas e médias e as últimas duas nas latitudes médias e equatoriais. Os exemplos da sua utilização podem ser encontrados em vários modelos regionais e de meso-escala $[1,4,7,8,13,15,17]$. Todas essas projeções podem ser tangentes ou secantes dependendo do tipo de interseção da esfera com o plano, cone ou cilindro.

Neste artigo consideraremos uma abordagem alternativa para geração de aplicações conformes da esfera para o plano. Ela é baseada na teoria de aplicações conformes planares e utiliza mais as propriedades analíticas das projeções do que as

\footnotetext{
${ }^{1}$ burstein@terra.com.br

${ }^{2}$ burstein@ufpel.tche.br
} 
geométricas. Essa abordagem permite classificar as projeções consideradas e revelar suas duas propriedades importantes. Na seção 2 daremos uma breve exposição dos resultados conhecidos de projeções clássicas estereográficas, cônicas e cilíndricas e das suas propriedades importantes para geração de grades computacionais. Na seção 3 introduzimos as aplicações conformes de esfera para o plano considerando a composição de transformações e estabelecemos a relação entre elas e projeções clássicas. A propriedade de independência do fator de mapa da longitude é investigada na seção 4 e, finalmente, na seção 5 representamos as transformações conformes de variáveis separáveis e a sua ligação com as projeções clássicas.

\section{Grades Computacionais para Regiões Esféricas}

As aplicações conformes estereográficas, cônicas e cilíndricas são bem conhecidos e utilizados muito tempo para representação da superfície da esfera em vários ramos da ciência, especialmente, em geodesia e cartografia, em geofísica, em ciências atmosféricas e oceânicas, em modelagem numérica. A descrição da teoria geral de transformações conformes e de projeções clássicas pode ser encontrada em vários livros relativos as ciências aplicadas (por exemplo [3, 6, 12, 14]), assim como nos livros de matemática $[5,10]$. Neste trabalho vamos nos referir principalmente ao livro de métodos matemáticos em cartografia de Pearson [11] e a uma revisão de geração de grades computacionais em modelos atmosféricos de Williamson [18].

Utilizando as coordenadas esféricas $\rho$ (raio), $\lambda$ (longitude) e $\varphi$ (latitude) podemos representar a projeção tangente estereográfica da esfera de raio $\rho=a$ para o plano com coordenadas polares $r, \psi$ na forma $[11,18]$

$$
\psi=\lambda, \quad r=2 a \cot \left(\frac{\pi}{4}+\frac{\varphi}{2}\right), \lambda \in[0,2 \pi), \quad \varphi \in\left(-\frac{\pi}{2}, \frac{\pi}{2}\right] .
$$

Essa projeção tem uma interpretação geométrica simples: as imagens são obtidas como pontos de interseção da reta, passando pelo pólo sul e ponto corrente da esfera, com o plano estereográfico.

A família de transformações conformes cônicas que tangenciam a esfera nos pontos da latitude $\varphi_{0}$ (do intervalo $\left.(0, \pi / 2)\right)$ são dadas pelas fórmulas $[11,18]$

$$
\psi=n \lambda, \quad r=a \frac{\cos \varphi_{0}}{n}\left(\tan \left(\frac{\pi}{4}+\frac{\varphi}{2}\right) / \tan \left(\frac{\pi}{4}+\frac{\varphi_{0}}{2}\right)\right)^{-n}, \lambda \in[0,2 \pi), \varphi \in\left(-\frac{\pi}{2}, \frac{\pi}{2}\right],
$$

onde $n=\operatorname{sen} \varphi_{0} \in(0,1)$ é o parâmetro que especifica o representante dessa família. As projeções cônicas não têm uma representação geométrica exata. No entanto, para entender melhor a forma dessa transformação pode ser utilizada uma interpretação geométrica aproximada. Ela consiste em construção de um cone geométrico tangente a esfera ao longo da latitude $\varphi=\varphi_{0}$ e com vértice localizado no eixo polar acima do pólo norte. As imagens dos pontos da esfera são os pontos obtidos na interseção do cone com a reta que passa pelo centro da esfera e o seu ponto corrente. Depois o cone é cortado ao longo de um dos meridianos e é desenrolado no plano. Nessa interpretação aproximada o parâmetro $n$ representa o ângulo do vértice do cone. Se assumir que $n$ igual ao este ângulo, então os valores de raio $r$ da 
transformação conforme e da sua interpretação geométrica são iguais somente nos pontos da latitude de tangencia $\varphi_{0}$.

Finalmente, a aplicação conforme cilíndrica tangente a esfera ao longo de equador tem a forma clássica $[11,18]$

$$
x=a \lambda, \quad y=a \ln \tan \left(\frac{\pi}{4}+\frac{\varphi}{2}\right), \quad \lambda \in[0,2 \pi), \quad \varphi \in\left(-\frac{\pi}{2}, \frac{\pi}{2}\right],
$$

onde $x, y$ são as coordenadas cartesianas do plano de projeção. A transformação cilíndrica também não admite uma representação geométrica exata. Numa ilustração aproximada, a esfera se coloca dentro do cilindro cujo eixo coincide com eixo polar da esfera e que tangencia a esfera nos pontos de equador. As imagens dos pontos da esfera se encontram utilizando a reta que passa pelo centro da esfera e seu ponto corrente. Depois o cilindro é cortado ao longo de um dos meridianos e é desenrolado no plano.

Para posteriores referências lembramos, também, das expressões do fator de mapa dessas transformações definido como a razão entre os comprimentos dos arcos elementares ao longo de curva plana e a curva esférica respectiva (ele também é chamado de fator de escala e de coeficiente métrico principal) [11, 18]:

$$
\begin{gathered}
m_{\text {str }}=\frac{2}{1+\operatorname{sen} \varphi}, \\
m_{\operatorname{con}}=\frac{\cos \varphi_{0}}{\cos \varphi}\left(\tan \left(\frac{\pi}{4}+\frac{\varphi}{2}\right) / \tan \left(\frac{\pi}{4}+\frac{\varphi_{0}}{2}\right)\right)^{-n}, n=\operatorname{sen} \varphi_{0} \\
m_{c i l}=\frac{1}{\cos \varphi} .
\end{gathered}
$$

Notamos que as aplicações estereográficas (tanto tangentes como secantes) podem ser obtidos como caso limite das transformações cônicas quando $n$ tende a 1 , mas estes dois grupos de projeções normalmente se consideram separadamente porque os primeiros têm fator de mapa definido no ponto correspondente ao pólo norte da esfera $(\varphi=\pi / 2)$ e os últimos não. Essa é uma peculiaridade importante para análise de aplicações e, portanto, falamos de três famílias de projeções.

Agora vamos definir os critérios de avaliação de grades computacionais construídas com base nas projeções conformes diferentes da mesma região limitada da esfera. Vamos considerar uma grade espacial ideal com o passo $h_{0}$ (homogênea no espaço físico do problema) e uma grade computacional com passo $h_{1}$ homogênea em coordenadas computacionais $(x, y)$. A aproximação real (física) é melhor (isto é, os passos físicos são menores) naqueles pontos da grade computacional onde o fator de mapa $m$ atinge os seus valores máximos $\left(m_{\max }\right)$ e é pior nos pontos dos valores mínimos $\left(m_{\min }\right)$. Assumindo que a precisão geral do esquema numérico é definida pelas regiões de maiores passos físicos, vamos precisar escolher o passo da grade computacional $h_{1} \approx h_{0} \cdot m_{\min }$ para garantir a aproximação equivalente ao passo físico $h_{0}$.

Uma outra característica importante do esquema numérico é a sua estabilidade. Para melhor entender a situação, podemos tomar como ponto de referência as equações de água rasa que é um modelo característico em dinâmica da atmosfera e oceano. Se considerarmos a aproximação dessas equações pelos esquemas numéricos 
que têm certo grau de explicidade, então o seu passo temporal máximo é determinado pela condição de estabilidade numérica (condição de Courant-Friedrichs-Lewy) que pode ser expressa na forma

$$
\tau_{\max } \approx \frac{h_{1}}{m_{\max } \cdot s}=\frac{m_{\min }}{m_{\max }} \cdot \frac{h_{0}}{s},
$$

onde $s$ é a velocidade de processos tratados de modo explícito. Por exemplo, $s$ é igual a velocidade de ondas gravitacionais para um esquema explícito (por exemplo, leapfrog); se utilizarmos um esquema Euleriano semi-implícito (por exemplo, esquema de Robert), então $s$ é igual a velocidade máxima do vento (velocidade dos processos advectivos); finalmente, se escolhermos o método semi-implícito semi-Lagrangiano, então $s$ representa a variação máxima da velocidade do vento $[2,9,16]$. Portanto, o número de passos temporais aumenta $\alpha$ vezes, onde $\alpha=m_{\max } / m_{\min }$, comparando com a grade física ideal (com $m \equiv 1$ ). Consequentemente, o mesmo esquema na grade computacional exige $\alpha$ vezes mais cálculos do que na grade ideal (onde $\alpha=1$ ). Se a forma das equações primitivas tem o mesmo grau de simplicidade em coordenadas diferentes, então o problema de otimização do sistema de coordenadas para geração de grade computacional consiste no encontro de tal projeção da esfera que assegura os valores mínimos de coeficiente de variação $\alpha$. Este é o caso das equações de água rasa (e em geral, das equações hidrodinâmicas tri-dimensionais) porque elas contêm a mesma estrutura tanto em coordenadas esféricas como em coordenadas cartesianas de qualquer projeção conforme.

Além disso, nos modelos de simulação real, incluindo os blocos físicos de parametrização de processos de sub-grade (tais como convecção vertical e condensação, turbulência e radiação), precisamos nos preocupar com a definição das escalas de sub-grade. Quando mais homogênea (em relação ao passo físico) for a grade computacional, mais homogênea será a definição das escalas de sub-grade e, portanto, mais confiáveis serão as parametrizações utilizadas. Portanto, tendo em vista tanto a eficiência do bloco dinâmico do modelo computacional, como a precisão do bloco físico, o problema de otimização da grade computacional para modelagem atmosférica e oceânica pode ser formulado como problema de minimização do coeficiente de variação $\alpha$ sobre a região esférica $\Omega$ em questão, isto é, minimização do seguinte funcional

$$
\alpha(m)=\frac{\sup _{\Omega} m}{\inf _{\Omega} m} \longrightarrow m \text { min }
$$

Obviamente, as duas projeções conformes cujos fatores de mapa diferem somente pelo um multiplicador constante são equivalente em relação ao coeficiente $\alpha$ e, portanto, geram as grades equivalentes. Esta é a situação que vamos encontrar freqüentemente nas próximas seções e a equivalência de tais projeções fica evidente, se observamos que um sistema de coordenadas pode ser obtido do outro pela simples mudança da unidade de medida para distância. 


\section{Composição de Transformações Conformes da Esfera para o Plano}

Primeiro desenvolvemos a construção alternativa de transformações conformes estereográficas, cônicas e cilíndricas que ajuda determinar algumas propriedades delas. Qualquer transformação conforme

$$
x=x(\lambda, \varphi), y=y(\lambda, \varphi)
$$

da esfera com coordenadas esféricas $\lambda, \varphi$ para o plano com coordenadas cartesianas $x, y$ satisfaz as condições de Cauchy-Riemann:

$$
\frac{\partial x}{\partial \lambda}=\cos \varphi \frac{\partial y}{\partial \varphi}, \quad \frac{\partial y}{\partial \lambda}=-\cos \varphi \frac{\partial x}{\partial \varphi} .
$$

Vamos considerar uma aplicação conforme arbitrária (3.1) como composição de duas transformações conformes: a primeira é uma transformação da região esférica para alguma região simplesmente conexa do plano e a segunda leva essa região a região final. Para garantir que a aplicação seja biunívoca, faremos um corte da esfera ao longo de algum meridiano, por exemplo, ao longo de $\lambda=2 \pi$. Na qualidade da região específica escolhemos uma faixa infinita do plano complexo estendido $\bar{C}$ :

$$
D=\{w \equiv u+i v \in \bar{C}: 0<\operatorname{Re} w<2 \pi n\},
$$

onde o parâmetro $n>0$ determina a largura dessa faixa. Neste caso, as fórmulas da transformação se encontram na forma

$$
u=n \lambda, \quad v=n \ln \left(\tan \left(\frac{\pi}{4}+\frac{\varphi}{2}\right)\right) .
$$

A aplicação (3.4) faz a esfera com corte $(\lambda \in(0,2 \pi))$ corresponder a faixa $D$ de tal modo que os meridianos da fronteira $\lambda=0$ e $\lambda=2 \pi$ correspondem a fronteira esquerda $(\operatorname{Re} w=0)$ e direita $(\operatorname{Re} w=2 \pi n)$ da faixa, respectivamente. As fórmulas (3.4) representam a aplicação cilíndrica clássica (2.3) $\operatorname{com} n=a$.

Aplicando a segunda transformação conforme

$$
z \equiv x+i y=f(w)
$$

que leva a faixa (3.3) na região final $\Omega=f(D)$, obtemos a aplicação da esfera primitiva com corte na região $\Omega$, que é conforme como composição de duas transformações conformes. O fator de mapa dessa projeção composta pode ser calculado pela fórmula

$$
m=\frac{n}{a \cos \varphi}\left|f^{\prime}(w)\right|, \quad\left|f^{\prime}(w)\right|=\sqrt{x_{u}^{2}+y_{u}^{2}} .
$$

De acordo com o modo escolhido para construção do conjunto de aplicações conformes (3.1), qualquer projeção cilíndrica está incluída nesse conjunto sob escolha do valor respectivo do parâmetro $n$ em (3.4) e sob definição da função $f(w)=w$ na segunda transformação (3.5). Obviamente, o respectivo fator de mapa é igual a $m=n /(a \cos \varphi)$. Caso escolhermos $n=a$, obtemos a projeção cilíndrica tangente (2.3). Para os valores $n<a$ obtemos as projeções cilíndricas secantes [11].

Podemos notar que a função linear

$$
f(w)=A w+B,
$$


onde $A, B \in C$, pode resultar geometricamente em translação, rotação e ampliação/contração uniforme de toda região (3.3) o que não influi nos valores de funcional (2.6). ). Assim, todas as projeções (3.4), (3.7) são equivalentes em termos de coeficiente de variação à projeção clássica (2.3).

Para gerar as transformações conformes estereográficas e cônicas, vamos considerar a função $f(w)=A e^{i w}, A \in R$, isto é $z=x+i y=A e^{i(u+i v)}$, onde $u, v$ são definidos pela fórmula (3.4). Então

$$
x=A \cos (n \lambda) \cdot\left(\tan \left(\frac{\pi}{4}+\frac{\varphi}{2}\right)\right)^{-n}, \quad y=A \operatorname{sen}(n \lambda) \cdot\left(\tan \left(\frac{\pi}{4}+\frac{\varphi}{2}\right)\right)^{-n}
$$

ou, em coordenadas polares,

$$
\psi=n \lambda, r=A\left(\tan \left(\frac{\pi}{4}+\frac{\varphi}{2}\right)\right)^{-n} .
$$

O respectivo fator de mapa é

$$
m=A \frac{n}{a \cos \varphi}\left(\tan \left(\frac{\pi}{4}+\frac{\varphi}{2}\right)\right)^{-n} .
$$

Se colocarmos $A=a \frac{\cos \varphi_{0}}{n}\left(\tan \left(\frac{\pi}{4}+\frac{\varphi_{0}}{2}\right)\right)^{n}, n<1$, então obtemos a projeção cônica clássica (2.2) com fator de mapa (2.5). Caso $A=2 a, n=1$, chegamos a projeção estereográfica (2.1) com fator de mapa (2.4). Podemos mostrar de maneira semelhante que as projeções (3.8) incluem também as projeções cônicas e estereográficas secantes (veja a definição das últimas em [11]).

Notamos de novo, que o uso da função mais geral

$$
f(w)=A e^{i \alpha w}+B
$$

onde $A, B \in C, \alpha \in R$ pode acrescentar apenas translação, rotação e ampliação/contração uniforme de toda região (3.3) o que não influi nos valores de funcional (2.6).

Resumindo, as funções (3.7) e (3.9) geram projeções cilíndricas, cônicas e estereográficas e, também, as projeções obtidas das últimas pelas transformações adicionais equivalentes em relação a coeficiente de variação $\alpha$, isto é, as de translação, rotação e ampliação/contração uniforme. Portanto, vamos nos referir às composições de (3.4) e (3.7) como às projeções cilíndricas e às composições de (3.4) e (3.9) como às estereográficas e cônicas.

\section{Independência da Longitude}

Agora vamos esclarecer qual é a posição das aplicações cilíndricas, cônicas e estereográficas entre todo conjunto de transformações conformes da esfera para o plano. Para isso consideremos as projeções conformes (3.1),(3.2) cujo fator de mapa (3.6) não depende da longitude $\lambda$. A última condição implica em

$$
\frac{\partial\left|f^{\prime}(w)\right|}{\partial \lambda}=\frac{\partial \sqrt{x_{u}^{2}+y_{u}^{2}}}{\partial \lambda}=n \frac{\partial \sqrt{x_{u}^{2}+y_{u}^{2}}}{\partial u}=\frac{n}{\sqrt{x_{u}^{2}+y_{u}^{2}}}\left(x_{u} x_{u u}+y_{u} y_{u u}\right)=0 .
$$


Essa equação pode ser reescrita na forma

$$
\operatorname{Re}\left(f^{\prime} \cdot \bar{f}^{\prime \prime}\right)=0 .
$$

Há dois tipos de soluções de (4.1). Primeiro, se $\bar{f}^{\prime \prime}=0$, então $f^{\prime \prime} \mathrm{e}$

$$
f(w)=A w+B, \quad A, B \in C .
$$

Caso contrário, equação (4.1) pode ser transformada a forma

$$
\operatorname{Re}\left(f^{\prime} \cdot \bar{f}^{\prime \prime}\right)=\operatorname{Re}\left(f^{\prime} \cdot f^{\prime \prime} \cdot \bar{f}^{\prime \prime}\right)=\left|f^{\prime \prime}\right|^{2} \operatorname{Re}\left(\frac{f^{\prime}}{f^{\prime \prime}}\right)=0 .
$$

Como $f(w)$ é uma função regular (isto é, função analítica univalente) e $f^{\prime \prime}(w) \neq 0$, então a função

$$
F(w)=\frac{f^{\prime}(w)}{f^{\prime \prime}(w)}=g(w)+i h(w)
$$

também é regular e, portanto, satisfaz as condições de Cauchy-Riemann:

$$
g_{u}=h_{v}, \quad g_{v}=-h_{u} .
$$

A equação (4.3) significa que $g(w)=0$ e, conseqüentemente, $h_{v}=0, h_{u}=0$, isto é,

$$
h(w)=\alpha_{1}=\text { const }, \quad \alpha_{1} \in R .
$$

Como $f(w)$ realiza transformação conforme, então $f^{\prime}(w) \neq 0, \forall w \in D$, isto é, $\alpha_{1} \neq 0$. Assim,

$$
\frac{f^{\prime \prime}}{f^{\prime}}=i \alpha, \quad \alpha=-\frac{1}{\alpha_{1}} \in R, \quad \alpha \neq 0
$$

e, portanto,

$$
\ln f^{\prime}(w)=i \alpha w+\beta, \quad \beta \in C
$$

ou

$$
f^{\prime}(w)=e^{\beta+i \alpha w}=A_{1} e^{i \alpha w}, \quad A_{1}=e^{\beta}, \quad A_{1} \in C, \quad A_{1} \neq 0 .
$$

Finalmente, obtemos

$$
f(w)=\frac{A_{1}}{i \alpha} e^{i \alpha w}+B=A e^{i \alpha w}+B, \quad A, B \in C, \quad A \neq 0, \quad \alpha \in R, \quad \alpha \neq 0 .
$$

Assim, todas transformações conformes com função de escala independente da longitude têm a forma (4.2) ou (4.4). O primeiro grupo representa as aplicações cilíndricas (3.4), (3.7) e o segundo é o conjunto de projeções cônicas e estereográficas (3.4), (3.9).

Notamos que a exigência a qualquer projeção da esfera para plano de ter fator de mapa independente da longitude é bem natural. Realmente, o problema de não homogeneidade da grade esférica ocorre devido a mudança do passo físico ao longo da latitude quando vamos na direção de um pólo ao outro (mudamos a latitude). Se deslocarmos na direção leste-oeste então esse passo não muda e o passo físico ao longo da longitude é o mesmo em qualquer local. Portanto é natural de buscar as soluções do problema da grade homogênea num conjunto de transformações que não mudam o espaçamento físico em relação a mudança de longitude, isto é, aqueles cujo fator de mapa não depende de $\lambda$. 


\section{Transformações Conformes Separáveis}

Nessa seção, vamos demonstrar a segunda propriedade analítica importante das transformações conformes estereográficas, cônicas e cilíndricas, a de separação. Utilizando as equações de Cauchy-Riemann (3.2) junto com a condição de separação

$$
x=x(\lambda), y=y(\varphi)
$$

obtemos as seguintes duas equações diferenciais ordinárias

$$
\cos \varphi \frac{d y}{d \varphi}=\frac{d x}{d \lambda}=n=\text { const }
$$

ligadas através da constante de separação $n$.

As duas equações (5.1) admitem soluções gerais na forma

$$
x=n \lambda+c_{1}, \quad y=n \ln \tan \left(\frac{\pi}{4}+\frac{\varphi}{2}\right)+c_{2},
$$

onde $c_{1}, c_{2}$ são duas constantes arbitrárias, $\lambda \in[0,2 \pi]$ e $\varphi \in(-\pi / 2, \pi / 2)$. Notamos que a escolha dos parâmetros $n>0, c_{1}=0, c_{2}=0$, que corresponde a uma escolha específica do sentido positivo do eixo $x$, e da origem das coordenadas planares, leva à projeção cilíndrica clássica (2.3). Obviamente, as projeções (5.2) têm o mesmo fator de mapa

$$
m=\frac{n}{a \cos \varphi} .
$$

para qualquer escolha dos três parâmetros e, portanto, todas elas são equivalentes à projeção clássica (2.3) em termos do coeficiente de variação $\alpha$.

Agora reescrevemos as condições de conformalidade de Cauchy-Riemann utilizando as coordenadas polares $r, \psi$ do plano de projeção:

$$
\frac{\partial r}{\partial \lambda}=r \cos \varphi \frac{\partial \psi}{\partial \varphi}, r \frac{\partial \psi}{\partial \lambda}=-\cos \varphi \frac{\partial r}{\partial \varphi} .
$$

Aplicando as condições de separação

$$
r=r(\varphi), \quad \psi=\psi(\lambda)
$$

obtemos

$$
-\frac{\cos \varphi}{r} \frac{d r}{d \varphi}=\frac{d \psi}{d \lambda}=n=\text { const },
$$

com coeficiente de separação $n$.

A solução geral de (5.3) tem a forma

$$
\psi=n \lambda+c_{1}, r=c_{2}\left(\tan \left(\frac{\pi}{4}+\frac{\varphi}{2}\right)\right)^{-n},
$$

onde $c_{1}, c_{2}$ são duas constantes arbitrárias $\left(c_{2} \neq 0\right)$. Caso pressupomos que $n>0$ (para sentido do ângulo $\psi$ coincidir com o de $\lambda$ ) e $c_{2}>0$ (porque $r$ deve ser positivo), a variação admissível de variáveis é $\lambda \in[0,2 \pi)$ e $\varphi \in(-\pi / 2, \pi / 2]$. Para parâmetros 
$c_{1}=0$ e $c_{2}=a \frac{\cos \varphi_{0}}{n}\left(\tan \left(\frac{\pi}{4}+\frac{\varphi_{0}}{2}\right)\right)^{n}, n<1$, a transformação (5.4) se torna a transformação clássica cônica (2.2). Para $c_{1}=0$ e $c_{2}=2 a, n=1$, obtemos a projeção estereográfica (2.1). O fator de mapa de (5.4) é igual a

$$
m=\frac{n}{a \cos \varphi} c_{2}\left(\tan \left(\frac{\pi}{4}+\frac{\varphi}{2}\right)\right)^{-n}
$$

e, portanto, todas as transformações (5.4) são equivalentes às transformações clássicas (2.1) ou (2.2).

\section{Agradecimentos}

Agradecemos à agência de pesquisa CNPq que apoiou essa pesquisa com a bolsa 300057/2004-0 e à agência de pesquisa FAPERGS que apoiou com a bolsa 02/0588-

7.

Abstract. In this study we develop one alternative approach to generation of conformal mappings from a sphere to a plane. The stereographic, conic and cylindrical projections, which are the most used ones in atmospheric and ocean modeling, are included in the set of considered mappings. Introducing the variation coefficient of the mapping factor as a measure of the homogeneity of computational grids, we use the proposed approach for classification of conformal mappings and for the proof of equivalence between the classes of the generate mappings and conformal mappings with mapping factor independent from longitude.

\section{Referências}

[1] R. Benoit, M. Desgagne, P. Pellerin, S. Pellerin, Y. Chartier e S. Desjardins, The canadian MC2: a semi-Lagrangian, semi-implicit wideband atmospheric model suited for finescale process studies and simulation, Mon. Wea. Rev., 125 (1997), 2382-2415.

[2] D.R. Durran, "Numerical Methods for Wave Equations in Geophysical Fluid Dynamics", Springer-Verlag, New York, 1999.

[3] H.R. Glahn, "Characteristics of Map Projections and Implications for AWIPS90”, TDL Office Note 88-5, National Weather Service, Washington, 1988.

[4] G.A. Grell, J. Dudhia e D.R. Stauffer, "A Description of the Fifth Generation Penn State/NCAR mesoscale model (MM5)", NCAR Tech. Note NCAR/TN398+STR, 1994.

[5] P. Henrici, "Applied and Computational Complex Analysis", Vol.3 Wiley \& Sons, New York, 1986.

[6] P. Knupp e S. Steinberg, "Fundamentals of Grid Generation", CRC Press, Boca Raton, 1993.

[7] L.M. Leslie e R.J. Purser, High-order numerics in an unstaggered threedimensional time-split semi-Lagrangian forecast model, Mon. Wea. Rev., 119 (1991), 1612-1623. 
[8] A.E. MacDonald, J.L. Lee e S. Sun, QNH: design and test of a quasinonhydrostatic model for mesoscale weather prediction, Mon. Wea. Rev., 128 (2000), 1016-1036.

[9] F. Mesinger e A. Arakawa, "Numerical Methods Used in Atmospheric Models", GARP Publications Series, Geneva, 1976.

[10] Z. Nehari, "Conformal Mapping", Dover Pub., New York, 1975.

[11] F. Pearson, "Map Projections: Theory and Applications", CRC Press, Boca Raton, II, 1990.

[12] P. Richardus e R.K. Adler, "Map Projections for Geodesists, Cartographers, and Geographers", North-Holland, Amsterdam, 1972.

[13] A. Robert, T.L. Yee e H. Ritchie, A semi-Lagrangian and semi-implicit numerical integration scheme for multilevel atmospheric models, Mon. Wea. Rev., 113 (1985), 388-394.

[14] J.P. Snyder, "Flattening the Earth: Two Thousand Years of Map Projections", The University of Chicago Press, Chicago, 1993.

[15] A. Staniforth, Regional modeling: A theoretical discussion, Meteorology and Atmospheric Physics, 63 (1997), 15-29.

[16] A. Staniforth e J. Côté, Semi-Lagrangian integration schemes for atmospheric models - A review, Mon. Wea. Rev., 119 (1991), 2206-2223.

[17] M. Tanguay, A. Simard e A. Staniforth, A three-dimensional semi-Lagrangian scheme for the Canadian regional finite-element forecast model, Mon. Wea. Rev., 117 (1989), 1861-1871.

[18] D.L. Williamson, "Difference Approximations for Numerical Weather Prediction Over a Sphere", GARP Publications Series, Geneva, 1979. 